waves moving in only one direction (as it may have seemed in Sec. III) - the motion of any relativistic string can be parametrized in a form closely analogous to the general solution of the (trivial) problem of transverse motion on a stretched string. If the $f_{i}$ and $g_{i}$ are small, they can be interpreted as transverse amplitudes of a string extending along the $x$ direction and the formal analogy takes on geometric life-both systems now actually look alike and evolve in time in similar ways. However, in contrast to the stretched string, the relativistic string can assume any arbitrary form (for example, it can intersect itself or even be closed) for suitably chosen $f_{i}$ and $g_{i}$.

\section{ACKNOWLEDGMENTS}

This work was supported by the David and Lucile Packard Foundation. V. E. also thanks Numo who originally posed the scattering problem several years ago.

\footnotetext{
${ }^{\prime} Y$. Nambu, Lectures at the Copenhagen Summer Symposium (1970). ${ }^{2}$ Y. Nambu, "Quark model and the factorization of the Veneziano amplitude," in Symmetries and Quark Models; proceedings of the International Conference on Symmetries and Quark Models, Wayne State University, 1969 (Gordon and Breach, New York, 1970), pp. 269-278.

${ }^{3} \mathrm{H}$. B. Nielsen, "An almost physical interpretation of the integrand of the $n$-point Veneziano Model," Contributed talk given at the 15th International Conference on High-Energy Physics, Kiev, 1970.

${ }^{4}$ L. Susskind, "Dual-Symmetric Theory of Hadrons I," Nuovo Cimento
}

A 69, 457-495 (1970)

${ }^{5}$ T. W. B. Kibble, "Topology of Cosmic Domains and Strings," J. Phys. A 9, 1387-1398 (1976).

${ }^{6}$ A. Vilenkin, "Cosmic Strings and Domain Walls," Phys. Rep. 121, 263-315 (1985).

${ }^{7}$ T. W. B. Kibble and N. Turok, "Self-intersection of Cosmic Strings," Phys. Lett. B 116, 141-143 (1982).

${ }^{8}$ P. Goddard, J. Goldstone, C. Rebbi, and C. B. Thorn, "Quantum Dynamics of a Massless Relativistic String," Nucl. Phys. B 56, 109-135 (1973).

${ }^{9} J$. Scherk, "An Introduction to the Theory of Dual Models and Strings," Rev. Mod. Phys. 47, 123-164 (1975).

${ }^{10}$ A. Vilenkin, "Cosmic Strings," Phys Rev. D 24, 2082-2089 (1981).

${ }^{11}$ C. Thompson, "Dynamics of Cosmic String," Phys. Rev. D 37, 283297 (1988)

${ }^{12}$ A. Albrecht and T. York, "Topological picture of cosmic-string selfintersection," Phys. Rev. D 38, 2958-2962 (1988).

${ }^{13} \mathrm{~T}$. Goto, "Relativistic Quantum Mechanics of One-Dimensional Mechanical Continuum and Subsidiary Condition of Dual Resonance Model," Progr. Theor. Phys. 46, 1560-1569 (1971); O. Hara, "On Origin and Physical Meaning of Ward-like Identity in Dual-Resonance Model," Progr. Theor. Phys. 46, 1549-1559 (1971); M Minami, "Plateau's Problem and the Virasoro Conditions in the Theory of Duality," Progr. Theor. Phys. 48, 1308-1323 (1972).

${ }^{14}$ M. Kaku, Introduction to Superstrings (Springer, New York, 1988), pp. 49-58; L. Brink and M. Henneaux, Principles of String Theory (Plenum, New York, 1988), pp. 97-149; M. Green, J. Schwarz and E. Witten, Superstring Theory: Introduction (Cambridge U. P., Cambridge, 1987), pp. 57-74.

\title{
Modeling games in the Newtonian World
}

\author{
David Hestenes \\ Physics Department, Arizona State University, Tempe, Arizona 85287
}

(Received 9 September 1991; accepted 19 March 1992)

The basic principles of Newtonian mechanics can be interpreted as a system of rules defining a medley of modeling games. The common objective of these games is to develop validated models of physical phenomena. This is the starting point for a promising new approach to physics instruction in which students are taught from the beginning that in science "modeling is the name of the game." The main idea is to teach a system of explicit modeling principles and techniques, to familiarize the students with a basic set of physical models, and to give them plenty of practice in model building, model validation by experiment, and model deployment to explain, to predict, and to describe physical phenomena. Unfortunately, a complete implementation to this approach will require a major overhaul of standard instructional materials which is yet to be accomplished. This article lays down physical, epistemological, historical, and pedagogical rationale for the approach.

\section{INTRODUCTION}

The great game of science is modeling the real world, and each scientific theory lays down a system of rules for playing the game. The object of the game is to construct valid models of real objects and processes. Such models comprise the core of scientific knowledge. To understand science is to know how scientific models are constructed and validated. The main objective of science instruction should therefore be to teach the modeling game.
If MODELING IS THE NAME OF THE GAME, it should be no surprise, considering the typical textbook, that students are perplexed and confounded by introductory physics. Students are left to extract the rules of the game for themselves from a torrent of details. That is akin to learning chess simply by watching the chessboard-pieces mysteriously disappearing and reappearing on the board, strange anomalies like castling, sudden terminations of play! The game is hard enough to fathom after you know the rules. 


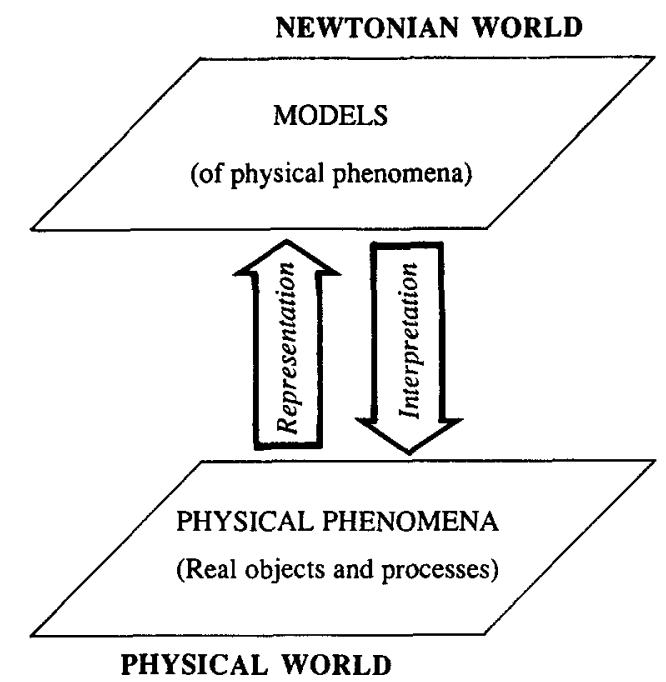

Fig. 1. Newtonian Epistemology. The Newtonian World is a conceptual world of possible models for actual objects and processes in the Physical World. Newtonian theory defines the range of possible models. Physical phenomena are interpreted (and so understood) by developing validated models to represent them.

No wonder that few students get the rules straight while the majority misses the point of the game entirely. Most students think the game is to collect and memorize facts. This blinds them to the beautiful structure of the Physical World revealed by science.

This paper aims to clarify the objectives of physics instruction by delineating the structure of Newtonian mechanics from a modeling perspective. The purpose is to facilitate the teaching of modeling skills as the primary transferable skills applicable throughout science. Such skills can be developed only within the context of a specific scientific theory. Historically, Newtonian mechanics has served as the paradigm of a scientific theory, and it still serves that role well today. Accordingly, we approach the Newtonian World as a conceptual arena where one learns to play the modeling games of science. Only by learning the Newtonian Game well can one understand its limitations and the rationale for the more refined games of modern physics.

\section{DEFINING THE NEWTONIAN WORLD}

Newtonian theory, like every other scientific theory, defines a Conceptual World. This world is populated with conceptual models of real objects and processes in the Physical World (Fig. 1). A sharp distinction should be maintained between the Newtonian World and the Physical World it characterizes. Many students and textbooks fail to do this. Consequently, it is widely believed that Newton's laws are inherent in the Physical World, just waiting to be discovered, like Columbus discovered America. On the contrary, as Einstein repeatedly emphasized, ${ }^{1}$ the laws of physics are "free creations of the human mind."

Newton's laws were invested to describe certain regularities in the motions of real objects. These regularities are, indeed, inherent in nature, but they could not be discovered without the invention of adequate concepts to describe them. It is no accident that they were not discovered before the "Age of Newton," though they had always been on display in "the Book of Nature" for everyone to see. The very conception of "Newton's laws" would not have been possible without a sequence of prior conceptual inventions, including the inventions of (1) Euclidean geometry, which defines the concept of space, (2) the concept of acceleration, first employed by Galileo in the analysis of motion, (3) analytic geometry, invented by Descartes to represent geometric curves by algebraic equations, and (4) differenial calculus, the mathematical invention of Newton and Leibniz which proved to be essential not only for formulating Newton's laws but for applying them as well.

One moral of all this is that conceptual invention and empirical discovery go hand-in-hand. One cannot discover what one cannot conceive. Likewise, students must become familiar with the Newtonian World before they can recognize reflections of the Physical World within it and use it as a conceptual tool for understanding the Physical World. It follows that a primary objective of introductory physics should be to help students enter the Newtonian World. ${ }^{2}$ Actually, the Newtonian World must enter the student, for it is a conceptual world which must be recreated in the mind of anyone who would know it. Each student must literally reinvent the Newtonian World in his/her own mind to understand it. That is a creative act of high order, so it is no wonder that students find it difficult, especially considering the fragmentary presentation of Newtonian theory in the textbooks.

Newtonian theory defines the Newtonian World, but it was never spelled out completely by Newton, and it has been greatly refined and extended by physicists since. For these reasons, a pious invocation of Newton's three laws of motion (in a canonized translation from the Latin of his original words or even in a modern paraphrase) is an inadequate formulation of Newtonian theory, though it is standard textbook practice. Educational research has established that most students can recite these laws but few understand them. It is high time that this textbook tradition be broken and replaced by a sharp, compact, coherent, and complete reformulation of Newtonian theory, if only to specify precisely what students must learn and so guide instructional design. My own attempt at such a reformulation is schematized in Fig. 2. It has been expanded and discussed at some length elsewhere, ${ }^{3}$ so I can limit my remarks here to its rationale and pedagogical import.

The first thing to note about the Newtonian World defined in Fig. 2 is that it is populated exclusively by (point) particles. Extended bodies in this world are all reducible to the particles that compose them. ${ }^{3}$ Point particles do not exist in the Physical World; they are conceptual objects created by Newtonian theory. They are defined by Newton's laws, which specify their properties. Figure 2 schematizes an expanded version of Newton's laws. The most notable addition is the Zeroth law, which specifies the primitive kinematical properties of position and motion, thus defining the Newtonian concepts of space and time (which of course had their origins in Greek geometry). The Zeroth law is tacitly taken for granted in conventional physics textbooks, presumably for the historical reason that Newton and his contemporaries did not recognize it as a physical law. There is no justification for such an omission in this day and age, since Einstein has shown that the most profound deficiency of Newtonian theory resides in the Zeroth law. ${ }^{3}$

It must be admitted that the Newtonian World defined 


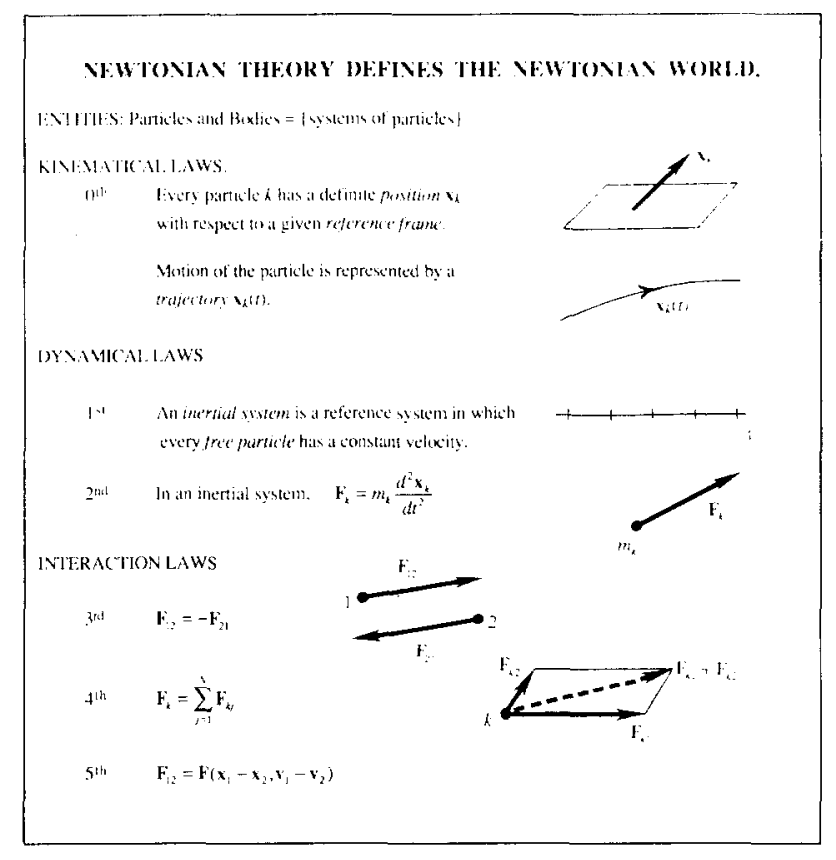

Fig. 2. Newtonian theory defines the Newtonian World.

in Fig. 2 is not quite the same as Newton's original conception; rather, it corresponds to the way Newtonian theory is understood and practiced by physicists today. Perhaps the greatest difference is the elimination of Newton's concept of "absolute space" in favor of relative position with respect to a specified reference frame.

The Zeroth law is the most complex and difficult of Newton's laws as well as the most fundamental. It provides the theoretical foundation for measurements of length, direction, and time, hence for all physical measurements. A full explication of the Zeroth law would include definitions of the concepts of rigid body, reference frame, and reference system, as well as their roles in measurement. Every measurement involves a comparison of objects or processes. Thus to measure the length of a given body is to compare it with a standard rigid body called a ruler, according to a well-known, well-defined procedure. Similarly, a measure of time is defined by the displacement of a "standard particle" called a clock moving with respect to a specified reference frame. On this ground, the concepts of velocity and acceleration can be defined and employed as quantitative descriptors for the motion of any particle compared to the clock. In this way the measurements of length, time, and motion are defined in the Newtonian World. Measurement in the Physical World is another story to which we return later.

With the concepts of space, time, and particle understood from the Zeroth law, the concepts of mass and force are defined by the rest of Newton's laws. In Fig. 2 I have reluctantly deferred to tradition in enumerating the First, Second, and Third of Newton's laws, but I have followed Arnold Sommerfeld in designating the superposition principle as a Fourth law independent of the others, and I have introduced a Fifth law to formulate crucial defining properties of force which are ordinarily introduced into Newtonian theory only tacitly.

More fundamental than a complete enumeration of Newton's laws is the threefold classification into kinemat- ical, dynamical, and interaction laws. This classification transcends Newtonian theory, applying to all of physics, indeed, to all of science. By employing it in Newtonian theory, we help prepare students for generalization to the rest of physics. Logically, the interaction laws should be placed ahead of the dynamical laws which presuppose them. These laws define the concept of interaction, expressed as the concept of force in Newtonian theory. Ironically, I regard the Fifth law, which has never before been counted among Newton's laws, as the most fundamental of the interaction laws, because it declares the existence of two particle force functions dependent only on the relative positions and velocities of the particles. The Fourth law (superposition principle) simply defines the net force on any particle as the vector sum of two-particle forces. All forces are therefore reducible to two particle forces.

The dynamical laws connect interactions with kinematics and so determine the particle motions. The subtle, almost superfluous, role of Newton's First law should be noted. A free particle is, of course, defined as one on which the net force is zero. This provides a criterion distinguishing inertial systems from other reference systems, and to say that free particles have constant velocities is to say that they define a uniform time scale. The definition of this time scale is an essential prerequisite to Newton's Second law. The First law has been previously classified as a kinematical law, ${ }^{3}$ but it is here classified as a dynamical law because it is an essential prerequisite to the Second law and it involves the concept of force.

To assert that the Newtonian World is defined by the complete set of laws in Fig. 2 is to assert that these laws are axioms which define the concepts of particle, motion, force, and mass. Definitions of force and mass have been matters of much unproductive dispute among physicists and philosophers, owing, I believe, to defective epistemologies and notions of definition. Let us be clear about these concepts. In the first place, it should be recognized that Newtonian theory rests on a constructivist epistemology which, as indicated in Fig. 1, maintains a sharp distinction between the (Real) Physical World and the conceptual Newtonian World constructed to describe it. Definitions pertain only to the conceptual world, not at all to the physical world. The purpose of a definition is to establish the meaning of a concept by specifying its relation to other concepts. When this has been done, we say that the concept is well defined. There are two ways to do it, yielding two kinds of definition: explicit and implicit.

A concept is defined explicitly by expressing it directly in terms of other concepts. This is the conventional notion of definition, used, for example, in defining "momentum" as mass times velocity. On the other hand, a concept is defined implicitly by specifying a set of axioms which relate it to other concepts. Thus the concept of a "geometrical point" is defined by the axioms of geometry, which specify its relation to other points, lines, and planes. Such axioms must be included in a full statement of the Zeroth law. Similarly, the concepts of "mass" and "force" are implicitly defined by the rest of Newton's axioms (laws) in Fig. 2. Axioms are statements employed to define concepts implicitly by specifying relations among them. Axioms are set apart from other statements or equations by accepting them as definitions, so they need not be proved, though it should be established that they are mutually consistent. New terms like "particle," "mass," and "force" which are 
supplied with meaningful relations by a system of axioms are commonly said to be "undefined terms." This is a seriously misleading expression that ought to be expunged, because it conflicts with established usage of the term "well defined." Terms are well defined by a complete set of consistent axioms. It is better to say that "some terms in a theory must be defined implicitly" rather than "some terms must be undefined."

Newton's laws function as axioms in defining the conceptual Newtonian World. But the term "law" signifies that they are more than axioms, they have physical interpretations that give them another dimension of meaning. A physical interpretation for a quantitative concept like "mass" or "force" can be introduced by specifying a procedure for measuring it. Such a measurement procedure is commonly called an "operational definition." I agree with Mario Bunge ${ }^{4}$ that this terminology is a serious abuse of language which should be avoided, as it invites confusion between the concepts of measurement and definition. A definition, whether implicit or explicit relates concepts to concepts, not concepts to things. The concept of measurement is something else, which we address later.

The concept of "operational definition" has its origins in the positivist epistemology most powerfully espoused by Ernst Mach in his famous critique of Newtonian mechanics. ${ }^{5}$ Mach held that physical laws are merely summaries of sensory experience and the meaning of physical concepts is determined only by specifying how they are related to experience. It is in this sense that "operational definitions" are intended to give meanings to physical terms. As a prime example, Mach presented a procedure for measuring the masses of particles in terms of their accelerations which is often presented as a typical operational definition today. ${ }^{6}$ On the contrary, our constructivist epistemology invites us to regard this simply as an application of Newton's axioms to the design of an experiment for measuring mass, which is possible only because mass is already well defined by Newton's axioms. In fact, Mach's procedure is not very practical. There are many other ways to measure mass, for example, by using conservation laws in collisions, and new procedures are continually being developed as physics evolves. All of them are useful, but none by itself provides a suitable "operational definition" of mass. It is the common definition of mass by Newton's axioms that interrelates the mass values obtained by the different procedures.

Einstein admired Mach greatly, but he opposed Mach's positivism vehemently, especially with his insistence that physical concepts are free creations of the human mind. Einstein's epistemological stance has come to be known as constructivism in recent years, ${ }^{7}$ and, of course, it is the stance adopted here. Positivism holds that the meaning of physical concepts is extracted from physical experience, whereas constructivism holds that meaning is constructed and matched with experience to make the experience meaningful and the meaning experiential. The processes of construction and matching are major components of the Modeling Theory discussed below. This presupposes the sharp distinction between the Conceptual World and the Physical World in Fig. 1. Constructivism acknowledges some truth in positivism, but only a half-truth.

Having identified the fundamental role in Newtonian Theory of the defining axiom system in Fig. 2, something can be said about its pedagogical significance. I submit that for a secure understanding of Newtonian mechanics, stu-
Table I. Rules for Newtonian modeling games.

Board: The three-dimensional Euclidean space of some physical reference system.

Pieces: Point particles or model objects composed of particles.

Objective: To produce validated models of material objects and processes in the Physical World.

Legal moves:

(1) Particles can be assigned any initial positions or velocities in the reference system consistent with specified interactions.

(2) Particles can be assigned any interactions consistent with the general interaction laws (Fig. 2).

(3) Particle trajectories must be calculated from the general dynamical laws (Fig. 2) or laws derived therefrom.

(4) A model is validated by matching it to physical phenomena in accordance with the defining laws (Fig. 2).

dents should become intimately familiar with the entire conceptual system in Fig. 2 (or any better version of it). They should know that the primitive concepts of space, time, particle, mass, and force are implicitly defined by this axiom system, and all other concepts in mechanics can be explicitly defined in terms of these primitives. Finally, they should know how these concepts are employed to model the physical world, a matter to be addressed in subsequent sections.

To help students see the defining axiom system as a whole, I have designed the schematic version in Fig. 2 as a single page to be handed out to students at an appropriate time. This could be supported by additional handouts providing full explications of each of the separate lawsknowledge which, in any case, should be readily accessible to the students. The icons to the right of the laws in Fig. 2 are not mere decorations, but cues to diagrammatic representations and physical interpretations of the concepts.

I do not advocate presenting Fig. 2 to students at the beginning of an introductory mechanics course, because they are not prepared to understand or employ it. Instead, I recommend that after studying kinematics they be given an explicated version of the Zeroth law to summarize and systematize their kinematical knowledge. After a survey of the various kinds of force, the interaction laws can be summarized. Finally, after Newton's Second law has been introduced, the complete axiom system can be presented.

\section{NEWTONIAN MODELING GAMES}

The Newtonian World is the conceptual arena where the Newtonian games are played. There are many such games, with various levels of difficulty, but they have the common objective of producing validated models of physical phenomena, and they share the same basic system of rules derived from the defining laws of Newtonian Theory. A concise unofficial version of these rules is given in Table $I$. Like the rules of chess, they specify the game board, the pieces, rules for moving the pieces, and winning the game. Beginners must know these rules before they can play Newtonian games with any assurance. Additional rules are required for specialized games.

There are two general classes of modeling games: theoretical and experimental. Few professional physicists are equally adept at games of both kind, or even all the games 


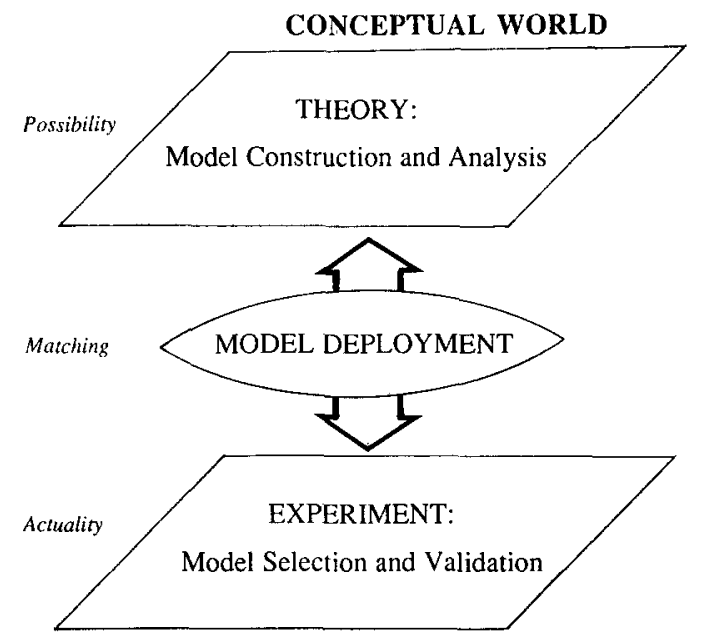

PHYSICAL WORLD

Fig. 3. Scientific Method can be described as a proces of generating conceptual models and selecting those which best represent physical phenomena. The theorist is primarily engaged in constructing and analyzing possible models for physical phenomena. The experimentalist is primarily engaged in detecting regularities in physical phenomena which can be interpreted by a conceptual model. Thus theory and experiment are complementary components of a single cyclical process for generating validated knowledge about the physical world, that is, scientific knowledge.

of one kind, so they must collaborate to play the more difficult games well. Generally speaking, theoretical games involve developing and analyzing models while experimental games involve evaluating models empirically and employing them in physical exploration. A model which has been shown to adequately represent phenomena in some empirical domain is said to be (empirically) validated. Clearly, both theoretical and experimental games must be played to produce validated models. As indicated in Fig. 3, the theoretical games are played entirely within the conceptual domain of the Newtonian World, whereas the experimental games are played in the physical world to articulate it with the conceptual world. The theorist builds the conceptual world (a world of the possible), while the experimentalist explores the physical world (the world of the actual).

\section{A. Theoretical games}

Theoretical games are of three general types: (1) model building, (2) model ramification, and (3) model deployment. In the first type, the objective is to build a model to meet given specifications, often derived from empirical data of observations. Historically, the first great game of this type was won by Newton himself, of course! Newton was challenged to find the force law governing planetary motion. Robert Hooke and others had guessed that it was an inverse square law, but they could not win the game because they did not have a complete set of rules. Though others had some of the rules, Newton was the first to assemble them into a coherent system; that involved inventing the differential calculus which was essential to formulating his Second Law of Motion. Then, with consummate skill he was able to derive the form of the gravitational force law from Kepler's three kinematical laws and reverse the argument. Thus he constructed and validated the very first model of a dynamical system: a point particle subject to a central gravitational force. Note that Newton's model was automatically validated by matching it to Kepler's laws, which already had a well-defined domain of empirical validity. Subsequent deployment of the model established that it had a much broader domain of validity.

Ramification games are played to analyze the properties (that is, the ramifications) of complex systems. Newton was drawn into such games as soon as he considered the superposition of gravitational forces on a single particle. For example, to justify his analysis of projectile motion Newton was induced to prove his famous theorem that the gravitational force due to a spherically symmetric earth is equivalent to that of a single mass located at the center of the earth. This, of course, raised questions about ramifications of more complex models for the earth which have occupied geophysicists to this day.

Newton devised a general strategy for ramification games which has been a mainstay of theoreticians ever since, albeit with continual refinements and elaborations. It is called linearization and perturbation theory. The idea is to expand interactions in some kind of power series. A model is said to be linearized when terms of higher order than the first are neglected. Linear models are amenable to exact mathematical analysis, though the complete mathematical theory is extensive. After a linearized model has been "solved," perturbation theory can be applied to evaluate corrections due to higher-order terms in the original expansion.

The full ramifications of nonlinear models are much more difficult to ascertain, as they involve such complex phenomena as phase transitions and chaos. Even the Newtonian three body problem, to classify the motions of three particles with mutual gravitational interactions, has not been fully solved, though it has engaged generations of physicists and mathematicians since Newton. ${ }^{8}$ Recently, however, computer simulation has developed into a practical method for studying the behavior of complex systems. Thus theorists have a new ramification game to play.

Deployment games involve the matching of models to empirical phenomena and data. Often a given model has already been validated in some empirical domain. Deployment of the model to account for new data from that domain is then expected to be routine. If a suitable match of the data to the model cannot be achieved, it may be suspected that the data are defective, possibly due to experimental error.

Deployment of a validated model in a new empirical domain may lead to genuinely new physical knowledge, as Newton was the first to discover, no doubt because he had the first opportunity. As every physics student should know, Newton applied his dynamical model of planetary motion to the moon and to projectiles near the surface of the earth, demonstrating not only that these diverse phenomena can be accounted for by the same basic model, but also establishing quantitative relations among them. Thus he generated evidence that his law of gravitational attraction is a universal gravitational force law.

The games of scientific explanation and prediction are two popular deployment games. A physical phenomenon can be explained by a theory only to the extent that it can be modeled within the theory. Thus the model is the explanation! Most explanations are only partial or qualitative. Often in a qualitative explanation no model is mentioned, though one is implied to someone who knows the theory. For example, an explanation like "the tides are due to 
gravitational attraction by the moon" makes no explicit mention of a model, but an explicit model is essential to explain such details as why the tidal period is half the period of the earth's rotation. In a prediction game, the model is usually more explicit, because it is needed to generate some trend in simulated data.

\section{B. Experimental games}

Experimental games can be classified as model deployment games. Deployment is the empirical component of modeling. Experimental deployment games differ from theoretical deployment games just mentioned in that their objective is to test and validate models. This formulation of the general objective of experimental activity requires some justification, lest it be dismissed as too narrow from either an experimental or a theoretical perspective.

Experimentalists might object that they are engaged in exploring the physical world for new phenomena, not merely evaluating models proposed by theorists. Experimentalists often underestimate the influence of theory even on their own activities, and positivism reinforces this tendency by claiming that theory is subservient to experiment. For example, Kammerlingh Onnes, the discoverer of superconductivity, declared that the only road to knowledge was via careful, persistent, systematic measurements, and he even questioned the value of Maxwell's equations as late as 1924. It seems he completely overlooked the role of theory in deciding what is worth measuring. How such a gross oversight might occur can be understood by examining the parochial training of some experimentalists even today. A young student can start working in a physics laboratory with very little background. Most likely he (or she) would be introduced to some new experimental technique. If he has the "good hands" of a "born experimentalist" he might perfect the technique and thereby gain some renown in his field. As there is no theory to be seen in his laboratory, he might regard his activities as theory independent. Since he is successful in an established experimental tradition, he needs no theoretical motivation and may remain oblivious to the theoretical rationale for his field. He did not like those theory courses anyway!

Fortunately, there are more perceptive experimentalists. They recognize that there is a critical theoretical component to the design of every piece of apparatus and everything they do in the laboratory, though much of the theoretical rationale has been forgotten and replaced by standard experimental procedures or it functions through qualitative mental models that guide experimentalists through mazes of apparatus to perform experiments on unseen objects. All this has been described in superb detail by Martin Deutsch, ${ }^{10}$ who notes
“...The experimental procedure may consist simply in noting the reading on the recorder as a function of the magnet current. Both the change made-the position of the control knob-and the resulting effect--the reading on the recorder-seem almost negligible in the to- tality of material involved in the experiment... . How is it possible that important and reliable conclusions are drawn from this experimenta- tion? The answer lies in the fact that the exper- imenter starts out with a well structured image of the actual connections between the events

taking place. Far from approaching the problem with the completely open mind which is supposed to be characteristic of the scientist investigating in an unbiased fashion all possible connections, he starts with the conviction that all of the relevant occurrences except the one which he is actually investigating in the experiment are either fully understood or at least in principle explicable on the basis of the preconceived image. Without this image, the experiment certainly could not have been conceived in the first place."

Experimental activity has two major components: (1) The design and interpretation of experiments, and (2) the collection and interpretation of data. Besides their intuitive role described by Deutsch, models play an essential formal role in both activities. Consequently, they are subject to the rules of the modeling game.

Consider first the collection and interpretation of data or measurement, as it is usually called. For Kammerlingh Onnes the refinement of measurement was the quintessential game of science. The most fundamental measurements in physics are measurements of length and time. The theory underlying such measurements is so well established and validated that it is seldom recognized as a theory at all. Even the fact that the theory needs empirical validation was not recognized before Einstein. An explicit formulation of this theory is none other than the Zeroth law (Fig. 2). The Zeroth law specifies the rules to be obeyed (the allowed moves, if you will) in making and comparing kinematical measurements of length and time. An operational analysis of procedures for measuring length has been made by Campbell. ${ }^{11}$ Despite being incomplete, it reveals some of the theoretical subtleties and complexities that are involved. Though the Zeroth law provides the theoretical foundation, many other physical laws are involved in modern practice to increase the range and precision of kinematical measurements. Electromagnetic theory is required for very large as well as very small scale distance measurements, while quantum theory is needed for precise time measurements with atomic clocks. There is no such thing as a physical measurement without a theoretical context!

The analysis and interpretation of data is as much a part of measurement as the procedures employed in data collection, for without them the data are meaningless. Data are meaningful only to the extent that they are related to some conceptual model. An experiment which does not contribute to the validation or invalidation of some model is useless, though the model may not be formulated before the experiment is performed. This crucial role of models in measurement is not often recognized.

Historically, the first great example of model deployment in data interpretation was Kepler's analysis of Tycho Brahe's data on planetary motion. Recall that Ptolemy employed the epicycle model to explain Greek data. The model was taken to be an explanation, because it was a composite of uniform circular motions, which Greek "theory" regarded as "perfect motions." Copernicus explained essentially the same data with the simpler model of uniform circular motion about the sun. His result does not look so revolutionary today, because we see the Ptolemaic and Copernican models as the same model represented in different reference systems. The real Copernican revolution was in recognizing the possibility of using a different ref- 
erence system and showing how to use it in data analysis. This possibility has since been recognized as a fundamental principle and formally incorporated into our formulation of the Zeroth law. Kepler employed the Copernican reference system in his own analysis and showed that Tycho's more accurate data could not be fitted to the Copernican model. This is a prime example of the importance of precision measurement emphasized by Onnes, but it also illustrates that models are essential to make measurements meaningful. Since no one before had ever considered any kinematical alternative to uniform circular motion, Kepler had to invent his own to fit the data. His brilliant result is formulated as a system of functional relations called $\mathrm{Ke}$ pler's laws. Many physicists would insist that Kepler's laws were discovered rather than invented. On the contrary, what Kepler discovered was that these laws fit the data. He had considered and discarded many alternatives. It would be better to speak of Kepler's model (rather than laws) and say that the model has been validated to the precision in Tycho's observations. We now know that many alternative models could be invented to fit the same data, but Kepler's is the simplest of all models in this class. We also know that Kepler's could not fit the more accurate data collected with telescopes rather than the naked eye, because the elliptical planetary orbits are perturbed by gravitational forces from other planets, as Newton was eventually to establish with his dynamical theory. That fact could never be discovered by Kepler's method; the more powerful method of Newton was needed. It is no small irony that Newton's law of gravitation would undoubtedly have been more difficult to discover if Kepler's model had been quickly invalidated by more accurate data. Here we have the possibility that scientific progress might be impeded by greater experimental precision. Onnes never considered that! On the other hand, Kepler would certainly not have invented and validated his model had not Tycho improved the methods and instrumentation, that is, the precision of astronomical measurement!

Experimentalists often look for patterns or regularities in their data which can be described by simple functional relations. This is often called "modeling the data." But that is a dangerous misnomer if "models" are regarded as representations of real objects and processes. An empirical relation is not a model. The crucial distinction between empirical relations and models is often overlooked or taken for granted. An empirical curve is meaningless without a model to interpret it. For example, Galileo's "law of falling bodies" $x=g t^{2} / 2$ refers to a model object in a particular context which includes the specification of a reference frame. Only for a specified model and reference system can the equation be understood as describing the "process of falling."

The dictum "let the data speak for itself" rests on the positivist conviction that meaning can be extracted directly from experience. In contrast, constructivism holds that theory generates models and equations to describe and explain, while experiment selects those models which match the phenomena. Data interpretation is the selection of a model, often from a family of models with adjustable parameters. It has been aptly said that the purpose of a scientific experiment is to ask a question of Nature. This being so, the answer is a validated model, not merely a mound of data.

To see how measurement fits into the general context of experimentation, we must consider the design and interpretation of experiments. We can distinguish two kinds of experimental questions or tests: those testing the validity of a scientific theory itself, and those testing the adequacy of a model derived from the theory. Let us discuss each kind in turn.

The defining axioms of Newtonian theory are called laws, because they have been empirically tested and validated in a broad empirical domain. That domain is so broad, in fact, that they were believed to be universally valid (or true!) throughout the eighteenth and nineteenth centuries. Only in the twentieth century have definite limitations of the validity of Newtonian theory been set by relativity theory and quantum mechanics. The axioms of the theory cannot be empirically tested either directly or independently. They can only be tested indirectly through their implications for model building. Only models can be tested experimentally, models of physical phenomena which can be studied experimentally. Thus theories are empirically validated only by validating models derived from them.

As an example, consider the validation of Newton's Third law, the only one of his three laws that Newton supported with experimental evidence when introducing it in his Principia. The experiments he mentioned were (tacitly) designed to test a model of two interacting particles. A good account has been given by Arons, ${ }^{12}$ so we can be brief. First, Newton cited momentum conservation in collision experiments as evidence that the Third law applies to contact forces. Though momentum conservation had been formulated before him from experimental evidence, only Newton could have deduced its relation to the Third law, because only he possessed the Second law to make the connection in a two particle model. Second, as evidence that the Third Law applies to forces acting at a distance, Newton described his own experiments on a system of two attracting magnets separated by a barrier. There he made the crucial observation that the system would be selfaccelerating if the internal (magnetic) forces did not cancel. Perhaps Newton's most convincing evidence for the Third law is in its striking implications for many interacting particles in Book 3 of the Principia; that included the new prediction that the tides on earth are jointly due to gravitational attraction by both the sun and the moon. However, he applied the Third law to modeling in Book 3 as if its universal validity were already beyond question. The results must surely have consolidated his confidence in the Third law. Indeed, the models Newton developed in Book 3 led to a host of new predictions; some were soon confirmed by observations of the Royal Astronomer John Flamsteed, while others kept astronomers busy for the next two centuries. Here is a prime example of how theories generate questions to be investigated by experiment or observation.

Newtonian theory is so well-validated today that empirical tests of its validity are no longer of scientific interest. Nevertheless, models of physical phenomena which have been developed according to Newtonian rules must be validated empirically to determine how adequately they describe the phenomena, because the models always involve assumptions not covered by the rules. No single model ever captures the full complexity of a real object. However, in principle there is no definite limit to the complexity which can be modeled. The trick is to select the simplest model 
which can account for the empirical data. This is Occam's razor expressed as a basic principle of model deployment. The simplest model for a physical object is, of course, a single particle. This is an adequate model if the dimensions of the object can be neglected, a criterion which can be evaluated both experimentally and theoretically. The theoretical evaluation is carried out by constructing a sequence of models with increasing complexity, so at each stage in the modeling sequence the model can be deployed to evaluate what was neglected in the previous stage. This is an important modeling strategy, which might be called the principle of successive refinements. It should not be surprising that the first great application of this principle is to be found in the Principia, because Newton was the first person to know all the rules of the modeling game. Let us recall what he did. ${ }^{13}$

Book 3 of the Principia (The World System) is Newton's crowning achievement. Here Newton played the modeling game with his gravitational force law and won by showing how to validate it as a universal law, the first known fundamental force law. His strategy was to develop and deploy a sequence of models. First, he modeled the planets as particles and solved the gravitational one body problem with a fixed center of force. Therefrom he derived Kepler's laws, the empirically testable consequences of the model. Next, he solved the two body problem and showed that this implied quantifiable deviations from Kepler's laws. Thus he established that Kepler's laws are not inherent in nature. Rather, they are testable properties of a simple dynamical model which are empirically observed under conditions where the model applies. Next, Newton formulated the many body problem, and, though he could not solve it, he was able to derive testable consequences therefrom. Thus he was able to deploy the model to explain observed "lunar inequalities" as due to perturbation of the moon's orbit by the sun and predict a perturbation of Saturn's orbit by Jupiter which was soon detected by Flamsteed. Finally, he moved from a particle model to an extended body model for the earth and developed his theory of the tides, which had enough empirical implications to keep astronomers busy for generations. Sad to say, even today few textbooks pursue this modeling game as far as Newton; Ref. 3 is an exception.

This case illustrates several general principles of experimental design which scientists have applied with consistent success since Newton. They are summarized by the following prescriptions.

(1) Select a physical phenomenon to investigate experimentally. Aside from experimental accessibility, the choice will be motivated by some theoretical issue, such as the functional form of the gravitational force law in the above case.

(2) Develop a model for the phenomenon from theory. Many models have free parameters which must be determined empirically. Also, as has been noted, it is often advisable to develop a sequence of models to provide theoretical estimates for the precision of testable consequences.

(3) Derive testable consequences of the model. "Testability" may depend on the available experimental apparatus or require improvements in instrumentation.

According to this account of experimental design, every theoretical aspect of modeling may be involved: model development, ramification, and deployment. However, in practice some of the steps are often skipped. A single ex- periment is seldom sufficient for investigating a significant physical phenomenon. So once the phenomenon has been selected a sequence of experiments may establish an experimental tradition without reconsidering the initial motivation for each experiment. Likewise, models developed early in an experimental tradition may be repeatably deployed with little modification to interpret experimental results. Such was the case, for example, with Newton's models for celestial mechanics. Consequently, within an experimental tradition the question of experimental design tends to be reduced to the single issue of improving the precision of measurements. When precision measurement becomes the sole end in itself, the tradition has become scientifically moribund. To survive, every experimental tradition needs the occasional infusion of the new theoretical issues and models.

Of course, there are variations of experimental design in different empirical domains. Astronomers speak of planning and interpreting their observations rather than designing experiments. The same general modeling principles are involved nevertheless. In astronomy, so to speak, the experiments have already been performed by Nature, so only the collection and interpretation of data is needed. The range of questions which can be asked of Nature was greatly extended by Galileo when he incorporated direct manipulation of the physical phenomena into experimental design. That was, perhaps, the greatest single advance in experimental science.

To wrap up this discussion of the many faces of modeling, an overview is schematized in Fig. 3, a variant of Fig. 1 which emphasizes the processes of modeling rather than the products, the models. As the figure caption suggests, our analysis of modeling provides the basis for a sharp formulation of Scientific Method, in noteworthy contrast to its vague formulation in most of the literature. It should be recognized that these modeling concepts generalize beyond mechanics to the rest of physics and, indeed, to all of science, though each scientific domain has specialized techniques for constructing and testing models. The general modeling concepts have been described here only in the domain of Newtonian mechanics, but this is especially fitting because that is where they originated.

\section{POPULATING THE NEWTONIAN WORLD}

The Newtonian World is populated by a rich variety of models developed by physicists engaged in Newtonian games. The games range in spirit from playful and practical to profound and serious. The physicist at play loves to explain "the how and why" of things. So it is that the pages of the American Journal of Physics are peppered with models to explain the surprising behavior of toys like the tippytop, the frisbee, and the superball. But in the main, the development of physics has been driven by a more serious concern ${ }^{14}$ known to philosophers since ancient times as the Search for Ultimate Causes.

Before Newton the search for ultimate causes made little headway. He transformed the search into a viable scientific research program when he replaced the vague notion of cause by his well-defined concept of force, so it became a search for fundamental forces. His program, laid out in the preface to his Principia, can be described as a modeling game; let's call it

NEWTON'S INTERACTION GAME: (1) From the motions of 


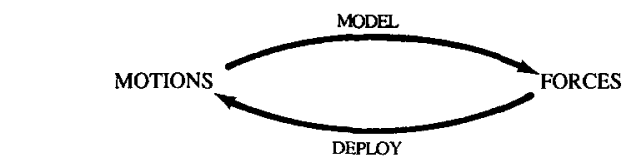

This reflects the general structure of Newtonian Theory (Fig. 2), as indicated by the diagram

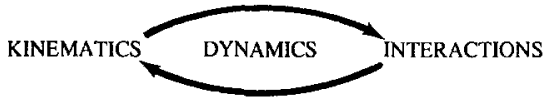

Fig. 4. Newton's Interaction game. The goal is to discover valid models for the interactions among material objects. The cyclical structure of the game is indicated by the diagram.

material objects model the forces on them, and (2) from the forces predict their motions in new situations (Fig. 4).

A win is scored by validating a new interaction model. The game, of course, is governed by the rules in Table I and Figure 2, and it is centered on the Fifth law, which asserts the existence of force functions, or force laws if you will. The object of the game is thus to formulate and validate specific force laws. As already noted, Newton himself scored the first great win with his "Universal Law of Gravitation." This spectacular success no doubt emboldened him to propose his game as a general approach to unravelling the secrets of Nature. The discovery of other fundamental force laws has not been so easy, however, so physicists are still playing the game, but with some new twists noted in the next section.

The interaction game is played at several levels, providing a basis for the classification of interaction models in Table II. The models range from phenomenological to fundamental. Nowadays, the development of a new interaction model is rare, but the deployment of such models is daily bread for physicists. Accordingly, a major goal of

Table II. A classification of interaction models. (The reader is invited to supply force laws modeling the enumerated interactions.)

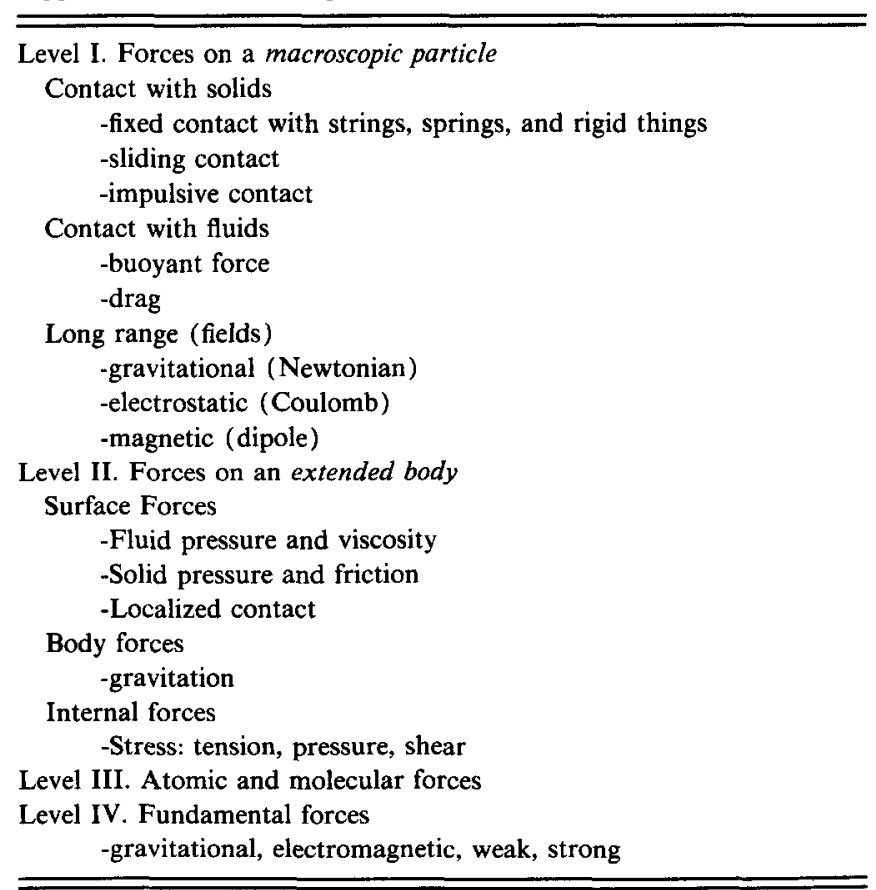

Table III. Basic models of material objects.

\begin{tabular}{ll}
\hline \hline Macroscopic models & \\
-point particle & -rigid body \\
-inextensible string & -elastic solid \\
-linear spring & -incompressible fluid \\
-ideal gas & -viscous fluid \\
Molecular models & \\
-Spring-lattice model of a crystalline solid & \\
-Particle models of a gas & \\
-Hard sphere model of a fluid & \\
\hline
\end{tabular}

physics instruction should be for students to become thoroughly familiar with the whole system of interaction models and how the models are deployed.

The search for ultimate causes has another aspect that was clearly articulated by Descartes. He suggested that all material objects are composed of irreducible parts (atoms) and that all change is merely rearrangement of parts; in other words, all change is reducible to motion. Undoubtedly this great idea helped convince Newton among others that the science of motion (mechanics) is the key to understanding Nature. Given the Newtonian theory that all change in motion is due to interactions, physicists have been led to the grand hypothesis that all properties of material objects emerge from interactions of simple irreducible constituents. This is a strong version of the so-called atomic hypothesis. It appears to be true in large measure, but physicists are still engaged in evaluating it. The evaluation enterprise is complex, because of the enormous variety of materials. Naturally, it can be described as a modeling game; let's call it

THE MATTER REDUCTION GAME: To derive the properties of material objects from the properties of their constituents.

Like the interaction game, this game is played at several levels leading to models of various types and complexity. Students must become familiar with the basic object models in Table III. Though they cannot be expected to invent such models, they must make the models their own by taking them apart to see how they work and deploying them in a variety of situations.

Of course, object models and interaction models must be deployed together, for interactions are properties of objects in the Newtonian World. Internal interactions are built into the models of extended objects, though in the simplest cases by merely imposing geometrical constraints. On the other hand, external interactions can be imposed on an object in an unlimited number of ways. The combination of an object model with model interactions is a DYNAMICAL MODEL. The variety of possible dynamical models is too broad to survey. Instead, a general strategy for developing such models is outlined in Table IV, which summarizes the distinctions made in Sec. III. Whole subtheories of mechanics are devoted to models of various types, including rigid body theory, continuum mechanics and fluid dynamics.

The development of dynamical models is the core of mechanics. Often, however, it is desirable to focus on some particular aspect of the models, as we did in classifying interactions. The same is done in classifying process models (Table V), which focus on changes in some particular property, usually one possessing a conservation law. It is 
Table IV. A general strategy for dynamical modeling games.

A. Model building

(1) Select the physical system and environment to be modeled. (The system can be any set or class of material objects.)

(2) Decompose the system into component parts which can be modeled as particles.

(3) Model the interactions of all components with one another (internal interactions) and with the environment (external interactions).

(4) Determine the equations of motion for the model system.

B. Model ramification, validation, and deployment

(1) Ramification: Analyze the behavior of the system by studying its equations of motion.

(2) Validation: Ascertain the empirical domain (including physical conditions and degree of precision) where the model applies.

(3) Deployment: Apply the model within its empirical domain to describe, explain and predict physical phenomena or design and interpret experiments.

important to note that the elementary kinematical models in Table V, including Kepler's, are of this type, for it is with these models that Newton's Interaction Game begins.

\section{BREAKING AND MAKING THE RULES}

Schrödinger described the game of science like this: ${ }^{15}$

"Science is a game-but a game with reality, a game with sharpened knives... . If a man cuts a picture carefully into 1000 pieces, you solve the puzzle when you reassemble the pieces into a picture; in the success or failure, both your intelligences compete. In the presentation of a scientific problem, the other player is the good Lord. He has not only set the problem but also has devised the rules of the game-but they are not completely known, half of them are left for you to discover or to deduce. The experiment is the tempered blade which you wield with success against the spirits of darkness-or which defeats you shamefully. The uncertainty is how many of the rules God himself has permanently ordained, and how many apparently are caused by your own mental inertia, while the solution generally becomes possible only through freedom from its limitations. This is perhaps the most exciting thing in the game. For here you strive against the imaginary boundary between yourself and the Godhead-a boundary that perhaps does not exist. You may indeed be given the freedom to unloose every bond, to

Table V. Process models and conservation laws.

\begin{tabular}{l}
\hline Particle kinematics \\
Constant acceleration, uniform circular motion, simple harmonic \\
motion, Keplerian motion \\
Rigid body kinematics \\
translation and rotation \\
Energy storage and transfer \\
Momentum transfer \\
Angular momentum storage and transfer \\
Change of phase \\
\hline \hline
\end{tabular}

make the will of Nature your own, not by breaking it or conquering it, but by willing it also."

The Ultimate Game of science is thus to discover the rules of Nature's Game, that is, to understand how the Universe works. God is the opponent, but, as Einstein has observed, $\mathrm{He}$ is not malicious though, $\mathrm{He}$ is subtle.

The goal of the Ultimate Game is too ambitious to be practical, so science has been subdivided into a medley of lesser games, each with the more limited objective of modeling some restricted domain of the Physical World.

Newtonian games are not the only games in physics, but all the others are variants. By skillful play of Newtonian games, physicists have been testing the rules to discover where they fail and trying out new rules to improve the game. The other great games of physics have emerged by this process of breaking and making the rules. It is enlightening to characterize these games by how their rules differ from Newton's rules set down in Fig. 2 and Table I.

\section{A. Maxwellian games}

Alexander Pope was mistaken when he declared "God said, 'Let Newton be, and all was light!' " In fact, there is no such thing as light in the Newtonian World of material particles. But that was rectified by Maxwell with his electromagnetic theory of light. Maxwell expanded the Newtonian World by introducing a new conceptual entity, the electromagnetic field, to be employed along with the material particle as a basic constituent of physical models. The first great victory in the new Maxwellian game was scored by Heinrich Hertz when he validated Maxwell's electromagnetic model of light waves.

\section{B. Einstein's relativity games}

Einstein recognized that Maxwellian electrodynamics does not quite fit into the Newtonian World, and he traced the problem to a flaw in the Zeroth law. The Newtonian version of the Zeroth law fails to accurately model the kinematics of objects at high relative velocities. Einstein rectified this by a subtle modification of the time concept in the Zeroth law. The Zeroth law is, in fact, a model of space and time. Minkowski showed that Einstein's modification changes the Zeroth law into a model of space-time in which there is no unique separation between space and time; thus, it is a fusion of geometry with kinematics. The games of Special Relativity are played in this new spacetime arena.

Einstein's General Theory of Relativity is a further modification of the Zeroth law to represent gravitation as a warping of space-time. It was his dream that all interactions might be reducible to geometry in a similar way. Physicists are still pursuing that dream.

\section{The quantum games}

Quantum Mechanics was developed because Newtonian theory failed to produce adequate models of very small objects like atoms and electrons. Quantum Mechanics does not tinker with the Zeroth law; Instead, it alters the basic concepts of particles, fields, and interactions. In other words, the Quantum Games are played on the same board with new pieces and different moves. The Quantum World is a new conceptual world populated with strange objects and peculiar processes. Many great victories have been 
scored in the Quantum Games, but the limits of the rules are still being tested.

Like Newton's games, all the great games of physics are modeling games, so they share all the general features of the Scientific Method schematized in Fig. 3: model building, ramification, deployment, and validation. Generic modeling strategies and tactics are effective in all the games. But each game has its own special modeling techniques, so great skill in one game does not necessarily transfer to equal skill in another.

\section{Beating the game}

Thomas Kuhn has suggested a distinction between Normal and Revolutionary scientific research which can be given a sharp and natural formulation in terms of modeling games. Normal Science plays the great modeling games strictly according to the rules. Revolutionary Science produces new games by revising the rules.

What happens to mavericks who do not want to play by the rules? Usually, they lose! They are ignored by their colleagues. History shows that winning by changing the rules is rare and achieved only by individuals who have mastered play by conventional rules. Evidently, only true masters can see where the rules fail.

\section{TEACHING THE GAME}

Conventional physics instruction is extremely inefficient. Educational research has established that students enter University physics with serious misconceptions about motion and force which are only moderately altered by the instruction in mechanics. ${ }^{16}$ The great majority of such students can state Newton's laws when they begin the course, but careful evaluation reveals that even at the end of the course they cannot consistently apply the laws correctly. Instead, the students' reasoning is still guided primarily by their intuitive misconceptions. Researchers have identified and categorized many such misconceptions, ${ }^{17}$ but two of them are particularly important, because they are persistent common sense alternatives to Newton's laws. Ignoring variations and nuances, ${ }^{17}$ these misconceptions can be formulated as intuitive principles.

I. The Impetus Principle: Force is an inherent or acquired property of objects that makes them move.

II. The Dominance Principle: In an interaction between two objects, the larger or more active object exerts the greater force.

Clearly, the Impetus Principle contradicts both the First and Second of Newton's laws, not to mention the Fifth, which requires that every force have an agent. The Dominance Principle comes from viewing interactions as conflicts in which the more powerful opponent is the winner, contrary to Newton's Third law. The presence of these misconceptions could be more serious, for they imply grave deficiencies in the concept of force, on which the whole of Newtonian mechanics depends.

One reason for the inefficiency of conventional instruction is a failure to take student misconceptions into account. A number of studies have achieved considerable improvement when misconceptions are dealt with explicitly and systematically. ${ }^{18}$ Nevertheless, the results fall short of what one would hope for, considering the apparently elementary and crucial concepts at issue. Misconceptions of Newton's Third law are especially recalcitrant. ${ }^{19,20}$ Though they may be found in as many as $90 \%$ of the students after conventional instruction, it has proved difficult to reduce the number below $60 \%$. It has often been suggested that such difficulties are due to the fact that the misconceptions are so deeply rooted in experience that we should not expect to alter them so easily. Our analysis in this article, however, suggests a different possibility.

Most attempts to eliminate misconceptions about force have dealt with them piecemeal, concentrating on individual misconceptions separated from the others. That approach ignores one of the most fundamental characteristics of the force concept, the coherence of the Newtonian theory. As explicated at length in Sec. II, all (six!) of Newton's laws are required to define the force concept. Thus the significance of Newton's Third law cannot be understood apart from its relation to the other laws. That relation is revealed only by applying the laws to construct and validate models of specific physical phenomena. This suggests a general strategy for dealing with misconceptions, elsewhere called "model-centered instruction:"21 Concentrate on teaching explicitly the principles and techniques of modeling with the Newtonian rules; this includes model validation for specific situations. In other words, teach the Newtonian modeling games. The instruction should be designed to elicit from the students explicit formulations of alternatives to Newtonian concepts to be analyzed and evaluated. In this way student misconceptions are confronted in specific contexts where a superior alternative is available. That is one of the primary conditions for conceptual change, a condition that is rarely met in conventional instruction. It should be recognized also that a comparison of plausible alternatives is an essential part of the validation process. Instruction in a physical theory without due consideration of alternatives is hardly more than indoctrination.

This general strategy for dealing with misconceptions within the context of modeling instruction can be implemented in many different ways. Malcolm Wells has employed it in the design of a laboratory based high school physics curriculum, which, in documented results published elsewhere, ${ }^{22}$ has proved to be exceptionally effective at eliminating misconceptions. One important conclusion from this and related work is that when instruction deals effectively with the most critical misconceptions, including the Impetus and Dominance principles, most of the other misconceptions tend to fade away without instructional intervention. This result is to be expected as students grasp the general force concept and integrate it into their thinking.

Strong historical support for the modeling approach comes from examining Newton's own conceptual development. Recent historical research has established unequivocally that during the two decades from Newton's initial research efforts to his writing of the Principia, Newton accepted the Impetus Principle as well as other misconceptions. ${ }^{23,24}$ From the early years he had at least rough versions of his three laws, derived largely from studying the work of others and modeling two particle collisions. ${ }^{25}$ However, he did not consolidate them into a coherent system with sharp formulations of all the laws until he was composing the Principia. There is little doubt that the event which precipitated all this was the challenge from Hooke and Halley to prove that the orbit of a planet subject to an 
inverse square force is an ellipse. Moreover, it was only when employing the Third law to establish the "universality" of his gravitational force law and model detailed dynamics of the solar system (as discussed in Sec. III) that the Third law was elevated to a prominent role in his thinking. Thus it was the activity of modeling that drove Newton to develop a coherent conceptual system and eliminate his misconceptions.

It was not some kind of intellectual inertia that kept Newton clinging to his mistaken belief in the Impetus Principle for 20 years of his professional life. It was the lack of a superior alternative or at least the conditions for developing one. When cognitive conflict was ignited (by Hooke's challenge), Newton's conceptual change was rapid and extensive. Similarly, a central problem of instruction is to establish optimum conditions for rapid conceptual change in students.

Concerning the crucial process of model and theory validation, empirical evidence (reviewed in Sec. III) that Newton adduced in support of his Third law is especially noteworthy. The usual way to teach the Third law is simply to present it as a rule to be memorized and require practice in applying it. ${ }^{19}$ The only justification is an implicit appeal to authority. No questions about validating it are raised. No wonder students are unimpressed. Why not adopt the Dominance Principle instead? Is it not more in accord with experience? Surely the Third law merits thorough justification both by establishing its coherence with the other laws and by examining critical empirical evidence.

\section{MASTERING THE GAME}

What does it take to master the modeling games in physics? Can just about anyone do it? Or does it take some special genius? Much can be learned about this by examining the intellectual lives of the master physicists. But first it must be recognized that untempered hagiology of these heroes in the textbooks and popular press has distorted their accomplishments out of all human proportion. As Einstein warned, we must be wary of "the general weakness of those, who, intoxicated with devotion, exaggerate the stature of their heroes."26 Acknowledging the social dimension of science, he remarked, "In science...the work of the individual is so bound up with that of his scientific predecessors and contemporaries that it appears almost as an impersonal product of his generation." From textbooks one gets the opposite impression that, for example, the whole science of mechanics was created by the single solitary genius of Newton. The danger in this is that it contributes to the alienation of science from society by purveying the impression that scientists are a breed apart with which few can identify.

What is this quality "genius" that raises the achievements of some scientists above that of their peers, if not an endowment of unique mental powers? A close study of the historical context and cognitive aspects of specific creative acts which are the hallmarks of genius suggests that the critical factor is possession of some special heuristic, that is, a unique conceptual approach to which others are not privy (at least, not until later) ${ }^{27}$ If this is right, then, what is the secret of Newton's genius? To answer that, we must first separate the evidence for Newton's general professional competence from the achievement that truly set him apart, namely, the creation of his Philosphiae Naturalis
Principia Mathematica. A sober historical evaluation leads to the conclusion that without the Principia-if Newton had perished before the age of 40 -he would be remembered as only one among many accomplished scientists rather than as a towering giant. ${ }^{25}$ In support of this conclusion, let us consider some of his other achievements.

\section{A. Newton's secret}

Newton was self-educated in mathematics, but from the beginning he had access to the most significant mathematical works of the time. He mastered seventeenth century mathematics within a year, ${ }^{24}$ but that consisted mainly of topics in analytic geometry and infinite series, so it does not surpass what university math students today are expected to learn within a year. More significant is the fact that Newton pursued the subject with extraordinary intensity and thoroughness, including developing from scratch a classification of 58 distinct types of plane cubic curves, all of which he plotted with care. The technical skill in analytic geometry which he acquired by such exercises set him far ahead of his contemporaries for the rest of his life.

Newton is credited with the invention of the differential and integral calculus. However, he was not the first to compute derivatives or integrals or relate the two by what is now called the fundamental theorem of calculus. ${ }^{28} \mathrm{New}-$ ton's chief contribution was to synthesize the many known examples into a general technique for differentiating or integrating any given function. This was truly a great achievement, but it was an inevitable climax in the mathematical climate of the day. It is likely that any number of other capable mathematicians could have done it. Predecessor James Gregory, who died at 36, was very close, and Wilhelm Leibniz actually did invent the calculus independently shortly after visiting England and learning some of the things about infinite series that Newton knew. Though Newton did it first, Leibniz published first, and he was more influential in subsequent development of the subject. If historians had spent less effort sorting out conflicting claims in the infamous priority dispute between Newton and Leibniz and more on determining the essential intellectual conditions for emergence of the calculus, we might have learned something about the creative process from the dispute. The longstanding tradition of assigning unique priority to individuals for key scientific inventions and discoveries has done much to mask the powerful social factors in scientific creativity. In fact, as in the case of calculus, multiple independent discoveries are more common than singletons, especially when they are important. ${ }^{29}$

In Newtonian hagiography there is much ado about Newton's intellectual fecundity in the famous year of the bubonic plague, 1665 , when he was 23 . The fact is, though, none of it would be of more than antiquarian interest, were it not for Newton's great reputation. Newton's "discovery" of the Universal Law of Gravitation is usually credited to that year, often embellished with the famous story of the dropping apple with its hidden metaphor of sudden inspiration. This is surely a myth, even without the apple, though it was encouraged by Newton himself well after the publication of the Principia, probably to push priority for "his law" back as far as possible to protect it from the vehement priority counterclaims of Hooke. He may have guessed the inverse square force law in 1665, just as Hooke did later, but he was in no position to prove it by establishing the connection to Kepler's laws. Besides his defec- 
tive understanding of dynamics, which we have already noted, his analytical techniques were still inadequate for that.

Newton's discovery of the binomial theorem in the same period is a good index of his growing analytical and pattern recognition skills, but it is hardly earth shaking and he never published it. His invention of the calculus is also placed in 1665, but we know from the nature of the subject that it cannot have been a sudden event. Though he probably conceived of his basic approach at that time, the evolution of his calculus continued for at least a decade. The first written account of his method was composed 4 years later, and it was another 2 years before his "overdot notation" for derivatives was adopted.

The last great accomplishment attributed to Newton in the "plague year" is the discovery that white light is a composite of the rainbow of colors. The fact is, though, that Newton took years to establish strong experimental support for his initial insight. ${ }^{24}$ Of greater moment, perhaps, was the precision of Newton's optical experiments in the subsequent years, which far surpassed what his contemporaries thought possible. From this we may surmise that he gained his keen appreciation of the great precision which can be expected of both experiments and mathematical theory.

A leading Newtonian scholar summarizes Newton's accomplishments in the famous year of the plague with these words: ${ }^{30}$

"When 1666 closed, Newton was not in command of the results that have made his reputation deathless, not in mathematics, not in mechanics, not in optics. What he had done in all three was to lay foundations, some more extensive than others, on which he could build with assurance, but nothing was complete at the end of 1666 , and most were not even close to complete. Far from diminishing Newton's stature, such a judgment enhances it by treating his achievement as a human drama of toil and struggle rather than a tale of divine revelation. 'I keep the subject constantly before me,' he said, 'and wait 'till the first dawnings open slowly, by little and little, into a full and clear light.","

As evidence of Newton's extraordinary powers, the story is often told about how he solved the famous brachristochrone problem after dinner one day, while Europe's mathematicians had been stumped on it for months. On seeing the anonymous solution John Bernoulli exclaimed, "I recognize the lion by his claw!" But how extraordinary is this performance, really, considering the fact that today a good undergraduate could be expected to solve the same problem for homework. True, the mathematical machinery at the student's disposal is highly perfected, but Newton had invented the calculus a quarter century before and spent years applying it to all sorts of gravitation problems. Moreover, the brachristochrone solution is a cycloid, and Newton was intimately familiar with this curve from Huygen's great work on clocks as well as from other sources. Consider also the report that Newton himself was stumped for years on a problem of only modest difficulty in the integral calculus, namely, to prove that the gravitational force due to a spherically symmetric object is as if all its mass were concentrated at its center.
With this background, let us turn to the critical question of what it took to create the Principia. I submit that Newton's Secret is twofold: (1) He was the first person to be technically proficient at applying the calculus to practical problems, and (2) he was the first to possess a complete and coherent system of principles for modeling the Physical World. In other words, he was the first person ever with opportunity to play the first great modeling game of science. And play he did! With demonic intensity! The Principia is the result!

Newton's greatest achievement was consolidating the rules of his modeling game. But mark how crucially this depended on his mathematical proficiency. We have already noted how the consolidation was finally achieved by modeling the Kepler problem and its two body generalization. This was a problem requiring all the correct rules, but simple enough to be mathematically manageable. Thus the rules of the Newtonian game were consolidated in the process of model construction and validation subject to the requirements of mathematical consistency. One might conclude that Newton was forced to invent the rules in order to play the game.

Once the rules were known, anyone with sufficient technical proficiency could play the game, as indeed many have done since with great success! The bulk of the Principia, then, is a record of the first person playing the game well. It was generated by the application of powerful technique rather than mysterious intuition. In this light Newton's creative feat may seem less prodigious. We marvel at the ingenuity of Newton's mathematical arguments, in part, because his methods were so unwieldy that no one since has fully mastered them. Before anyone could improve on Newton's performance, the world had to wait half a century for the development of better mathematical tools and techniques, primarily by Euler.

What can we surmise from this invasion of Newton's intellectual territory? I submit that the significance of Newton's priority is less than usually supposed. After "the secret of his success" became known, others could match him in mechanics and even surpass him in some respects. The creation of classical mechanics was inevitable, even without Newton, driven by attempts to create mathematical models of physical phenomena which were underway before Newton appeared. The emergence of classical mechanics would have been temporarily delayed if Newton had not appeared, but all would have been in place anyway for the emergence of quantum physics in the twentieth century.

None of this is intended to diminish Newton's achievements, but only to recognize them as the product of a universal human creative power which was not unique to Newton. That power is nothing other than an ability to create, interpret, and employ models of the Physical World. As we have seen in the example of Newtonian mechanics, that power can be enormously increased by welldesigned mathematical tools and modeling techniques. Before considering how these insights can help us with instruction, it will be helpful to place them in a more general perspective.

\section{B. Constructivist epistemology}

To analyze the cognitive processes of knowing and learning, we must imbed them in a suitable epistemological framework. The two piece epistemological model in Fig. 1 


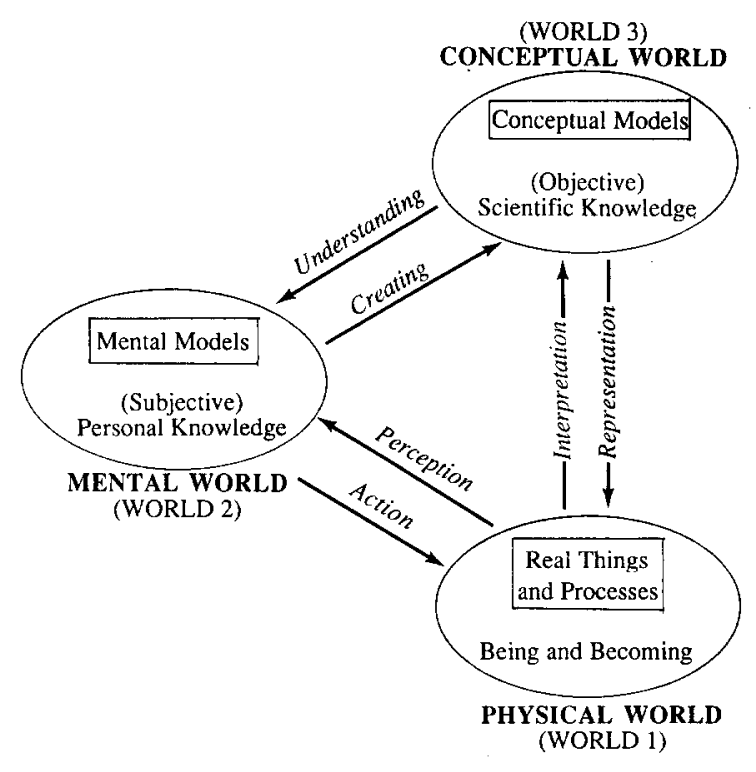

Fig. 5. Constructivist epistemology maintains that knowledge of the Physical World is achieved by constructing models of physical phenomena. The objective conceptual models constructed by the cooperative activity of scientists are distinguished from mental models constructed in the minds of individuals. Labels on the interactions between the three Worlds are meant to be suggestive rather than definitive.

is inadequate for this purpose, because the crucial role of the human mind is omitted. So we generalize it to the tripartite model schematized in Fig. 5. This is essentially the same as the three World model of Popper and Eccles, ${ }^{31}$ though we employ it here for a different purpose. In their terminology, World 1 designates the Physical World. World 2 designates the human mind of a single individual. World 3 designates the world of shared human knowledge called culture, though we limit it here to the subculture of science, the reservoir of scientific knowledge. It is a Conceptual World consisting of the shared concepts composing scientific knowledge. These concepts are objective in the sense that they are independent of any particular individual, though there is no such thing as a concept apart from someone to think it.

We interpret Fig. 5 as a constructivist model. There are many brands of constructivism, but they share the basic tenet that knowledge is constructed rather than discovered. In the version favored here, the basic constructive activity is making models of physical phenomena, but a sharp distinction is drawn between mental models in individual minds and the conceptual models of science. Conceptual models originate from mental models created in the minds of individual scientists, but they are given objective formulations which makes them independent of their originators. Conversely, an individual understands a conceptual model only by creating a mental model to represent it. The models are related by similarity, but the correspondence is rarely, if ever, a simple isomorphism. Typically, the mental model has irrelevant features peculiar to the individual's mode of thinking, or, in the mind of an experienced scientist, it is integrated into a rich and complex knowledge structure far exceeding the explicit range of the conceptual model.

Constructivist theory has at least two major implications of great pedagogical importance. First, it implies that understanding is a creative act. As Feynman expressed it in advice to himself on his last blackboard, "What I cannot create, I do not understand." 32 This means that understanding Newtonian theory is a creative act of high order, comparable to Newton's original creation. Newtonian theory cannot simply be transmitted like a TV image; it must be created anew in the student's mind, and only the student can do it. To drive the creative process, the student has the advantage of more powerful conceptual tools and stronger hints than Newton did. But Newton also had to draw on hints and tools from his predecessors and contemporaries. It appears, in fact, that mechanics could not have been invented before Newton's time, because the intellectual prerequisites were not sufficient. Newton had to develop the last of those prerequisites, the calculus, for himself.

This constructivist perspective should engender great respect for the creative powers of all students as well as for the difficulties in learning physics. It also informs us that active engagement (and not mere passive compliance) is essential for conceptual change. Emphasis is on the act! Of course, as we have already noted, the context in which the act takes place is equally important.

A second major implication of our modeling version of constructive theory is that pattern recognition skills are essential to understanding physics. Models can be regarded as patterns which are similar in some way to patterns found in the Physical World. In a mathematical model the pattern is expressed in mathematical form. Indeed, mathematics has been called The Science of Patterns. ${ }^{33}$ The fundamental role of pattern recognition skills in understanding and applying mathematics is not as widely appreciated as it should be.

\section{Pattern recognition in chess and physics}

The identification and analysis of specific pattern recognition skills involved in modeling and mathematics is a difficult research task for cognitive psychology on which not much progress has been made. However, studies of cognition in chess have produced insights with broad applicability. ${ }^{34}$ Experienced chessplayers have developed "perceptual vocabularies" of significant piece configurations and combinations (patterns) which they perceive as units and employ in evaluating positions and planning moves. Such a vocabulary, rather than brute computation, is the basis of insight into chess. For the best players (grandmasters), the size of the vocabulary has been estimated at more than 50,000 -about the same size as the word vocabulary of an erudite college professor. The question of greatest pedagogical interest is not the details of the vocabulary but how it is learned or, rather, constructed.

In physics as in chess, the brain will develop a vocabulary of patterns spontaneously from the experience of playing the game and studying examples of good play. There are two big differences, however, due to the fact that physics is so much more complex than chess. First, the chess player's attention is automatically confined to the chessboard where the patterns are to be found. But in physics a student who hardly knows what the game is about is likely to attend to the wrong things, thus overlooking the significant patterns and even learning the wrong ones (misconceptions). Therefore, control of student attention is crucial to good instructional design. The burden of this article is that student attention should be directed at models and modeling processes where the significant patterns are to be 
found. Lacking systematic attentional control, student learning under conventional instruction is hit or miss (mostly miss)!

A second difference from chess is that patterns in physics can be given many different representations (e.g., mathematical, graphical, or diagrammatic). Good pedagogical design is needed to control the choice of representation to enhance learning.

At least one more important thing about instructional design can be learned from chess. I offer some of my own observations from tournament chess. Thousands of chess players in the United States play organized tournament chess regularly and have official numerical ratings of their performance maintained and published by the United States Chess Federation. Amateurs and professionals are ranked together on the official Elo rating scale, which ranges from a few hundred for a beginner to about 2800 for the leading grandmaster. The title "master" is conferred on players with ratings above 2200 . This number is a traditional dividing line between amateurs and professionals, though many amateurs play at master strength and a professional must achieve a much higher rating to be successful. Less than $1 \%$ of the players fall into this category.

The records of Elo ratings contain a wealth of information about cognitive skills, but I offer only a few informal observations which I believe would be confirmed by systematic numerical evaluation. The ratings are remarkably good predictors of tournament performance and so are good measures of cognitive skill. Ratings of most tournament players rise fairly quickly when they first start playing regularly to a level which stays relatively constant for the rest of their lives, fluctuating with a standard deviation which I estimate as less than 100 Elo points. That skill level is maintained even after long periods away from chess or periods of intense tournament activity. An obvious question is, why does the skill growth level off so abruptly, and why is there no evident improvement with experience thereafter? A plausible answer is that after the pattern vocabulary is large enough for routine play, further growth is not induced by the demands of play; the old patterns suffice. This is supported by the observation that players who become masters continue to improve over a longer period. A 10-year period is typical for the development of grandmaster strength. The secret of this continued growth appears to lie in their approach to the game. Many players with obvious ability do not continue to develop so. The secret of those who do can be seen in the practice of postmortem analysis, which is widespread among professionals. Immediately after the game the opponents sit down together at a chess board to review the entire game while it is fresh in their minds-discussing strategic and tactical themes, analyzing promising alternatives at critical points, trying to identify losing moves and so forth. This is an ideal activity for inducing conceptual change-discovering weaknesses (bugs, misconceptions) in old thinking patterns and better patterns to replace them. It is an excellent way to promote the ability for what Piaget calls reflective thinking, namely, the ability to critically analyze ones own conceptual presuppositions and thinking patterns.

Post-mortem analysis has been explicitly incorporated into the instructional method of Malcolm Wells mentioned earlier, and it is certainly one of the keys to his success. In his laboratory-based curriculum, performing an experiment, collecting data, and all that is only the first half of a laboratory activity. The second half, to which equal time is allotted, consists of a post-mortem of the first half involving the entire class of about 24 students. This is probably when the most significant learning takes place, but it must be designed and directed with skill.

Another place where systematic post-mortem analysis would improve instruction is in problem solving. The homework submitted by most students shows little evidence of the reflective thinking which is essential to a high level of skill development. Even graduate students are satisfied with the most perfunctory solutions to problems, anything that arrives at the coveted "answer." But the opportunity for deepest learning is post-mortem analysis after the problem has been solved, addressing such questions as "What was the key to the solution? Can the argument be simplified? How can the result be verified? Are there other ways to solve the problem? Which is the best? Can the problem and solution be generalized? Of what interest is the result?" Such questioning is characteristic of the reflective thinking of professional physicists. Perhaps it is so rare among students because so little is done to promote it.

Reflective thinking is essential for mastering the technical skills of physics. Practice is not enough, for without the guide of reflective thinking it is likely to be misdirected. The significance of technical mastery should not be underestimated. We have already noted how critical it was to Newton's great achievement. Dating back to Newton, Cambridge University has a long tradition promoting the mastery of mathematical skills and producing great physicists-Stokes, Maxwell, Raleigh, and many others. Feynman's advice to himself on his last blackboard included: ${ }^{32}$ "Know how to solve all the problems that have been solved." His point is surely that a master theoretical physicist must master the successful reasoning patterns in his field. Where does one find these patterns? When the brilliant Norwegian mathematician Niels Henrik Abel was asked how he forged so quickly to the front rank, he replied, ${ }^{35}$ "By studying the masters, not their pupils." When Newton was asked how he discovered the universal law of gravitation, he replied, "By thinking on it continually!"36 His powers of concentration were, indeed, exceptional. Great skill and great accomplishments do not come easy.

A discussion of skill development and its pedagogical implications should not avoid the issue of innate talent. Newton's rapid intellectual development at about the age of 23 is often presented as evidence of his natural genius. However, I believe that such rapid development is a fairly common phenomenon. It is certainly common in the biographies of famous physicists and mathematicians-always regarded as a mark of genius. But no one has bothered to study how common it is in the lives of scientists with lesser reputations. I have noted it several times among graduate students, most recently in a meteoric rise to excellence by a student whom I initially thought was hopelessly mediocre. Quantitative estimates of the phenomenon in chess could be obtained from Elo rating data. From my own knowledge of the data, I would guess that it may occur in as many as $1 \%-10 \%$ of the chessplayers, depending, of course, on the criterion for a rapid rise. This may indicate some special talent for chess. However, we know that for the rise to be sustained to a high level of competence, favorable conditions are necessary and it must be driven by 
reflective thinking and intense effort. Talent is a prerequisite, but how much is sufficient? Again we get a clue from chess.

Here are my own observations, but I have found them in agreement with opinions expressed by strong masters in conversations and published remarks. There seems to be a natural dividing line among chess players near an Elo rating of about 2000 -officially designated as the expert level and reached by only a few percent of tournament players. The play of those above this level appears to be qualitatively different from that of most below. The difference can be described as a kind of strategic sense, and it is easily recognized by masters in the selection of moves a player makes. Players who possess this strategic sense-perhaps everyone ranked above 2000 - probably have the ability to approach the grandmaster level of play with sufficient dedication and training. But players without this strategic sense seem unable to cross or even approach the expert level no matter how hard they try. Of course, some players with the strategic sense have only a moderate (but never low) rating because they have not expended the concentrated effort needed to reach the expert level. Strategic sense can be enhanced by experience and training, but it evidently has an innate basis, because it can be recognized in the play of novices, even when they are quite young. Anecdotal evidence suggests that a master surveying the play of novices can quite reliably identify those who could develop into good chess players.

If competent physics research requires a strategic sense comparable to the one in chess, as I believe it does, we can apply the conclusions from chess to make comparable estimates of the fraction of students capable of research in physics. I will refrain from publishing my own guesstimate. We know so little, really, about scientific talent. Probably our schools are more effective at suppressing than developing it. We do know, though, that there are many deficiencies in conventional physics instruction. Only by expending the enormous effort in educational research and development required to eliminate them will be able to discover just how effective instruction can be and make reliable estimates of the reservoir of physics talent.

In the meantime, let us note that, though few chessplayers have the ability to become grandmasters, nearly all of them can play a credible game and appreciate the achievements of the grandmasters. Physics instruction should at least be able to raise the average physics student to a comparable level of competence and appreciation.

\section{CONCLUSIONS}

Most beginning physics students are wasting time playing the wrong game. They think the game is to collect facts and memorize procedures. This makes them blind to the structure of physics and its insights into the structure of the Physical World.

The recommended remedy is "model-centered instruction." Students should be taught from the beginning that the game of physics is to develop and validate models of physical phenomena. They should understand that a physical theory like Newtonian mechanics is a coherent system of design principles for constructing such models; in other words, a theory is a validated set of rules for playing modeling games. Students must develop a repertoire of models which have become intimately familiar by analyzing how they work and deploying them in a great variety of phys- ical situations to describe, to predict, to explain phenomena or to design experiments and devices. The models must be made their own, in the sense that the models are integrated into their thinking as conceptual tools for understanding physical phenomena. Playing modeling games should help them learn the objectives and methods of science.

Model-centered instruction can be implemented in many different ways. Richard Hake and Arnold Arons have been outspoken advocates of the Socratic method in physics instruction. ${ }^{2}$ In this method the instructor is not a source of information but the moderator of discussion among the students who stimulates the discussion with probing questions to induce students to articulate, clarify, criticize, and justify their beliefs. The method has at least two major strengths which should be incorporated into any instructional program: (1) It shifts the locus of control from teacher to student, making students responsible for their own beliefs and judgments. It is student centered rather than teacher centered. (2) It encourages reflective thinking, leading students to insights into their own thinking processes. In short, it promotes intellectual independence. The pure Socratic method, however, has serious weaknesses: It is not systematically directed at specific objectives, and it lacks a mechanism for introducing new ideas and conceptual tools to improve the quality of discourse.

For this reason a modified Socratic method is to be preferred, in which the instructor introduces ideas and evidence to enhance and guide the discourse. But this must be done carefully, lest it interfere with the nurturing of student independence. To be optimally effective, Socratic sessions must have coherence over an entire course. Early on, the students must come to an understanding on criteria for posing questions, formulating answers, and evaluating evidence. Model-centered instruction provides a coherent framework for all this which, as explained in this article, includes an explicit formulation of the scientific method. The instructor can stimulate the integration of this framework into the students' thinking by asking such questions as: "What is the physical system of interest and what interactions does it have with its environment? What model shall we adopt to describe the system? Which properties of the system are modeled and which are neglected or ignored? How well does the model account for the data?" The intent is to promote insight into the structure of scientific knowledge and argument, and to help students make it their own. Malcolm Wells has used this method successfully in post-mortems to lab activities. Postmortems should be a regular feature of student modeling games.

This article is intended as a contribution to instructional theory $^{21}$ grounded in a constructivist epistemology. As a foundation for model-centered instruction, Newtonian theory, and applications have been expressed in game formats which, hopefully, will help engage student interest and sharpen insight into the structure of the subject. We have reviewed a broad range of physical, epistemological, historical, and pedagogical rationale for this approach. However, the value of the instructional theory in guiding instructional design is yet to be proved. The vicissitudes of implementation cannot be discussed here. We only note that an early, incomplete implementation ${ }^{37}$ and a high school version by Malcolm Wells ${ }^{22}$ have produced encouraging results, but much more must be achieved before any 
flag waving is in order. Ideally, this article will attract some readers who can not wait for someone else to do the job.

\section{ACKNOWLEDGMENT}

This work was partially supported by a grant from the National Science Foundation.

'A. Einstein and L. Infeld, The Evolution of Physics: The Growth of Ideas from Early Concepts to Relativity and Quanta (Simon and Schuster, New York, 1938).

${ }^{2}$ R. Hake, "Promoting student crossover to the Newtonian world," Am. J. Phys. 55, 878-884 (1987).

${ }^{3}$ D. Hestenes, New Foundations for Classical Mechanics (Kluwer, Dordrecht/Boston, 1987; 4th printing with corrections, 1992), Chap. 9. It might be argued that the restriction of Newtonian Theory to particles as primitives is too restrictive for the purposes of continuum mechanics. Granted! However, particle mechanics can go a long way toward continuum mechanics, and it is much simpler and so more suitable for beginning students. The development of continuum mechanics only really got started a century after Newton with the work of Euler and Cauchy. A satisfactory formulation of continuum mechanics requires too much mathematical sophistication to address here.

${ }^{4}$ M. Bunge, Philosophy of Physics (Reidel, Dordrecht/Boston, 1973). Physics teachers can benefit from Bunge's incisive critiques of many physical and philosophical concepts.

${ }^{5} \mathrm{E}$. Mach, The Science of Mechanics: $A$ Critical and Historical Account of its Development, translated by T. J. McCormack (Open Court, New York, 1960), 6th ed.

${ }^{6}$ A. B. Arons, A Guide to Introductory Physics Teaching (Wiley, New york, 1990), p. 50ff.

${ }^{7}$ For example, R. Driver, "Theory into Practice II, A Constructivist Approach to Curriculum Development," Development and Dilemmas in Science Education, edited by P. Fensham (Falmer, London, 1988), p. 133-149.

${ }^{8}$ Reference 3, pp. 398-418.

${ }^{9}$ M. Dresden, H. A. Kramers (Springer-Verlag, Berlin 1987), p. 475.

${ }^{10} \mathrm{M}$. Deutsch, "Evidence and Inference in Nuclear Research," in Evidence and Inference, edited by D. Lerner (The Free Press, New York, 1957), pp. 96-106. See also Ref. 4, p. 77.

${ }^{11}$ N. R. Campbell, Foundations of Science (Dover, New York, 1957).

${ }^{12}$ Reference 6, pp. 64-68.

${ }^{13}$ I. B. Cohen, "Newton's discovery of gravity," Sci. Am. 244 (March) 166-179 (1981). This superb article demystifies Newton's greatest discovery by describing its origins, and it shows how Newton played the modeling game with a constructivist epistemology.

${ }^{14} \mathrm{D}$. Park, The How and the Why, An Essay on the Origins and Devel- opment of Physical Theory (Princeton U.P., Princeton, NJ, 1988).

${ }^{15}$ W. Moore, Schrödinger, Life and Thought (Cambridge U.P., Cambridge, 1989), pp. 348.

${ }^{16}$ I. Halloun and D. Hestenes, "The initial knowledge state of college physics students," Am. J. Phys. 53, 1043-1055 (1985).

${ }^{17}$ I. Halloun and D. Hestenes, "Common sense concepts about motion," Am. J. Phys. 53, 1056-1065 (1985). This article contains a fairly complete taxonomy of misconceptions about mechanics. See also Ref. 22.

${ }^{18}$ See, for example, Ref. 2.

${ }^{19}$ D. Maloney, "Rule-governed approaches-Newton's third law," Phys. Educ. 19, 37-42 (1984).

${ }^{20} \mathrm{D}$. E. Brown, "Students' concept of force: the importance of understanding Newton's third law," Phys. Educ. 24, 353-358 (1989).

${ }^{21}$ D. Hestenes, "A modeling theory of physics instruction," Am. J. Phys. 55, 440-454 (1987).

${ }^{22}$ D. Hestenes, M. Wells, and G. Swackhamer, "Force concept inventory," Phys. Teacher 30, 141-158 (1992).

${ }^{23} \mathrm{M}$. Steinberg, D. E. Brown, and J. Clement, "Genius is not immune to persistent misconceptions: conceptual difficulties impeding Isaac Newton," Int. J. Sci. Educ. 12, 265-273 (1990).

${ }^{24} \mathrm{R}$. Westfall, Never at Rest: A biography of Isaac Newton (Cambridge U.P., Cambridge, 1980).

${ }^{25}$ J. Herival, The Background to Newton's Principia: A Study of Newton's Dynamical Researches in the Years 1664-1684 (Oxford U.P., London, 1965).

${ }^{26}$ A. Einstein, quoted in E. B. Cohen, Revolution in Science (Harvard U.P., Cambridge, MA, 1985), Chap. 28, p. 439.

${ }^{27}$ D. Hestenes, "Secrets of genius," New Ideas Psychol. 8, 231-246 (1990).

${ }^{28} \mathrm{C}$. Boyer, A History of Mathematics (Wiley, New York, 1968), Chap. XIX.

${ }^{29}$ R. Merton, "Multiples and singletons," In The Sociology of Science (Univ. of Chicago, Chicago, 1973).

${ }^{30}$ Reference 25, p. 174.

${ }^{31} \mathrm{~K}$. Popper and J. Eccles, The Self and Its Brain (Springer-Verlag, Berlin, 1981)

${ }^{32}$ R. Feynman, "Feynman's office. The last blackboards," Phys. Today 42(2), 88 (1989).

${ }^{33}$ L. Steen, "The science of patterns," Science 240, 611-616 (1988).

${ }^{34} \mathrm{~W}$. Chase and H. Simon, "The Mind's Eye in Chess," in Visual Information Processing, edited by W. G. Chase (Academic, New York, 1973).

${ }^{35}$ E. T. Bell, Men of Mathematics (Simon and Schuster, New York, 1937), p. 308

${ }^{36}$ Reference 24 , p. 105.

${ }^{37}$ I. Halloun and D. Hestenes, "Modeling instructions in mechanics," Am. J. Phys. 55, 455-462 (1987).

\section{POYNTING'S THEOREM, ENERGY DENSITY, AND BEAUTY DENSITY}

The interpretation just given [of Poynting's theorem] suggests several comments. In the first place, the present authors are not able to ascribe any significance whatever to the phrase "localized energy." They do not believe that "Where?" is a fair or sensible question to ask concerning energy. Energy is a function of configuration, just as the beauty of a certain black-and-white design is a function of configuration. The authors see no more reason or excuse for speaking of a spatial energy density than they would for saying, in the case of a design, that its beauty was distributed over it with a certain density. Such a view would lead one to assign to a perfectly blank square inch in one portion of the design a certain amount of beauty, and to an equally blank square inch in another portion a certain different amount of beauty.

Max Mason and Warren Weaver, The Electromagnetic Field (Dover, New York; reprint of 1929 edition, University of Chicago, Chicago, IL), pp. 266-267. 